\title{
3D CITY MODELLING OF ISTANBUL BASED ON LIDAR DATA AND PANORAMIC IMAGES - ISSUES AND CHALLENGES
}

\author{
G. Buyuksalih ${ }^{1}$, P. Baskaraca ${ }^{1}$, S. Bayburt ${ }^{1}$, I. Buyuksalih ${ }^{1}$, A. Abdul Rahman ${ }^{2}$ \\ ${ }^{1}$ BIMTAS, Istanbul, Turkey - (gb@bimtas.istanbul, pbaskaraca@bimtas.istanbul, serdarbayburt@gmail.com, \\ ibuyuksalih@yahoo.com) \\ 23D GIS Research Lab, Universiti Teknologi Malaysia - alias@utm.my
}

KEY WORDS: 3D city modelling, Airborne LiDAR, Panoramic imagery, LoD2, LoD3

\begin{abstract}
:
This paper describes the generation of 3D city modelling of LoD2 and LoD3 buildings based on 3D point clouds data and other auxiliary data for Istanbul city, Turkey. The project is being undertaken by Istanbul Greater Municipality (IBB) since October 2012. The aim is to provide 3D information to the relevant city planning departments within IBB. The development of the 3D city model utilized several data acquisition techniques, software and computing tools as part of the methodology. The tools include from Riegl, TerraSolid, TerraScan, FME Workbench, MicroStation, and other visualization tools. The generated 3D city models illustrate how the high-resolution point clouds and 3D modelling play major role in such development. This paper also highlights several issues and challenges of the development, i.e. from data acquisition, processing of point clouds and the 3D modelling of buildings.
\end{abstract}

\section{INTRODUCTION}

Several major cities around the globe are developing 3D models of their cities and urban areas with the aim of using the models for several applications such as for planning, infrastructure, tourism purposes, etc. Cities like Berlin, Lyon, Wien, Rotterdam, Brisbane, New York, Stuttgart, Singapore are some of the examples (Kolbe, 2017), Khoo and Soon (2016), Stoter (2016) and Prandi et al (2015). City of Istanbul in Turkey also has initiated such 3D model mainly for city planning purposes (Buyuksalih, et al., 2018). This paper describes the development of 3D city model based on data captured from airborne LiDAR (Light Detection and Ranging) and panoramic images. This paper also highlights some issues and challenges associated to the development. Airborne LiDAR technology has become an effective tool for the use in applications of urban areas. In the field of urbanization, the LiDAR measurements can provide high-quality data on three-dimensional characterizations of buildings. A total of $5400 \mathrm{~km}^{2}$ airborne LiDAR data were collected for the city of Istanbul with Riegl Q680i scanner during the years 2012 and 2014. The flight campaign was carried out at an average flying height of $500 \mathrm{~m}$ by a helicopter at 80 knot flight speed. As a result of bundle adjustment, in total, approximately 17,000 LAS files with the file size of $500 \mathrm{~m}$ by $700 \mathrm{~m}$ have been generated for the whole city. The main object classes such as ground, building, vegetation (low, medium, high) were derived from these LAS files using the macros in TerraSolid software. After quality check for the classified point cloud, the 3D building cubes (approximately 1.5 million) were obtained automatically using TerraModeller software as a $3 \mathrm{D}$ CAD files with the contributions of ortophotos and building footprints available in the $1 / 1000$ scale base maps while some specific structures such as mosques, churches and bridges which they have complex roof type were manually generated. Then, these files were converted to the CityGML format using an FME Workbench. Finally, CityGRID software was used to get the topologically and semantically correct $3 \mathrm{D}$ models of the buildings. The creation of the LoD2 city model of Istanbul is time consuming and faces some difficulties. We utilize Unity3D software for visualization of 3D models. It provides 3D manipulation, simulation, light rendering of objects through functions defined using programming languages. However, LoD2 city model of whole Istanbul cannot be easily imported to the Unity environment due to some constraints - it has some issues and challenges. Besides all these, panoramic photography is being increasingly used and emphasized in recent years. $360^{\circ}$ panoramic photography is the tool that allows interactive static imaging where the viewer is no long a spectator but able to manipulate the objects in the scene (dynamically). Istanbul city has embarked on the new method of $3 \mathrm{D}$ data collection that is panoramic imagery. Acquisition of $360^{\circ}$ Panoramic and gigapixel images of Istanbul provides the integration of all these imageries to the Web applications served by IMM (Istanbul Metropolitan Municipality). Providing easy access to all streets, many cultural, historical and touristic places of Istanbul via Web-based services (both in Android and IOS platforms) and available on VR glasses. Integration of the laser device to the mobile mapping system enables 3D laser point cloud with camera shots and can be used to rectify these images and create a base data for measurements on the image. Thus, precise detail measurements on images are possible and these images could be used as a measurable photo. By combining airborne and mobile data, more accurate and precise data could be achieved and thus, generate better information for architectural designs, urban planning, solar energy applications, silhouette applications, and other environmental-based projects. This capability enables the LoD2 city model to be upgraded to the LoD3 by the integration of the $360^{\circ}$ metric-panoramic imageries. The Istanbul project creates innovation and potential for more advanced research in the domain

The remaining of the paper describes 3D city modelling in Section 2. Data collection, processing and 3D modelling in Section 3. Section 4 describes the complete workflow of the 3D city modelling generation from data acquisition to LoD2 and LoD3 objects. Section 5 highlights some issues and challenges, and finally conclusions in Section 6.

\section{3D CITY MODELLING}

3D city models are often used to make predictions such as exploration, analysis and synthesis on city data in relation to the future. The most important feature of 3D urban models is that 
different spatial information can be combined and displayed in the same environment. It also allows for the creation and management of complex urban models. 3D city models are frequently used in architectural design, presentation and evaluation of the city. Urban planning is complex and multidimensional tasks since urban spaces should be planned, considered and studied in 3D environment. Examples of 3D environments, including, 3D virtual city models, terrain models, building models, plant models, roads, and also geographic-based city data. For 3D buildings, scale concept is expressed in the level of detail of the LoD (Level of Detail). Each LoD shows a specific level of generalization. Unlike 2D topographic maps of specific scales, there are no generally accepted LoDs for 3D buildings as standard. Currently, LoD is mostly based on data resolution, content of semantic information and applications. 3D city modelling with the level of detail also contributes to the minimization of the costs of obtaining and using 3D spatial data. Comprehensive rules for 3D urban modelling have not yet been established. In order to facilitate the sharing of 3D building data, standards have been developed by the OGC (Open Geospatial Consortium), that is the CityGML (City Geography Markup Language) modelling language, see reports from OGC (2012, 2018). It is an open data model and XML-based standard for the representation of $3 \mathrm{D}$ urban objects. It is an OGC standard realised for the storage and exchange of virtual 3D city models and covers broad thematic fields of city objects - geometrical and topological aspects can be accurately described and linked with their semantic part. CityGML 2.0 has some basics features as reported by OGC and also by researchers like Biljecki (2017). The basic descriptions of LoDs (Biljecki et al, 2014 and Stoter, 2016) are: LoD0 - the digital terrain model. Aerial photo or map of the relevant models can be associated with the digital elevation model; LoD1 - the model shows box-like buildings with some heights. It is the simplest level of 3D building models. At this level of detail, the buildings are shown as rectangular with flat roofs. LoD2 - is a model created by adding building roofs to the level of LoD1. At the level of LoD2, building roof types, exterior details and plants are shown in a certain proportion. LoD3 - LoD2 with the outer texture of the exterior. At this level of detail, it is created with architectural models with properties such as balconies of buildings, wall details, roofs. High resolution photographs are placed on the outer surfaces of structures at this level of detail. Detailed plant models and portable objects are also shown at this level. LoD4 - LoD3 detail level structures with objects inside the building such as rooms, stairs, interior walls, furniture (see Figure 1).

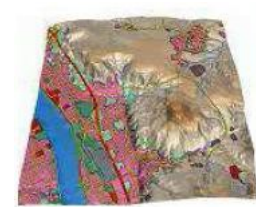

LODO
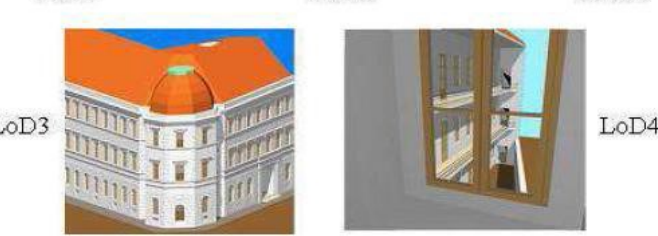

Figure 1: The 5 LoDs (after Kolbe, 2017)

Based on the LiDAR points cloud. buildings are vectorized as DGN files. These files together with orthoimages and digital elevation models are used for the generation of a 3D city model. The 3D city model generation is mainly based on the software solution from UVM Systems. Nevertheless, the transformation from DGN files to XML files uses the FME workbench. The unavoidable topologic mistakes, as double lines and snapping mistakes (overshoots and undershoots) are corrected in most cases automatically, but a visual check is required including the fit in relation to the used height model which defines the lower end of the buildings. The UVM Systems software includes mainly the CityGrid Modeler and the CityGrid Builder for editing and visualization.

\subsection{The Istanbul 3D City Modelling}

The Istanbul city 3D model utilises LiDAR data that covers an area of $5400 \mathrm{~km}^{2}$. The data collection system is an integrated one (digital camera, GPS, IMU and other direct sensor navigation devices etc.). Georeferenced LAS data, digital surface model (DSM), digital elevation model (DEM), normalized digital surface model (nDSM), ortho-images and LoD2 level 3D urban model were created (see Figure 2). Various classifications, automatic and manual corrections were made until the 3D urban model was obtained with the desired details.

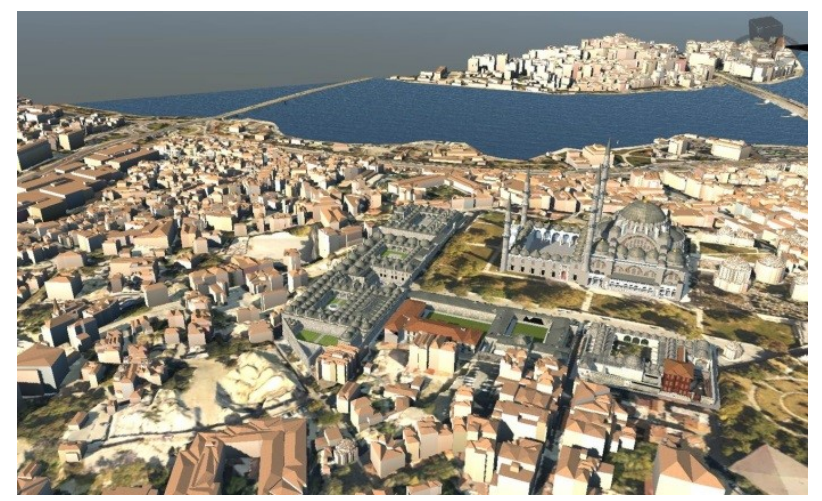

Figure 2: Major landmarks as LoD3 (mosques)

\section{DATA ACQUISITION, PROCESSING AND 3D MODELLING}

\subsection{LiDAR Data Acquisition}

Laser scanner, camera, IMU (Inertial Measurement Unit) and the GPS receiver are integrated with an aircraft mounted laser sensor, and their signals are compensated by data from GPS signals, IMU and ground stations to obtain an intense, high-accuracy data set. The strategy for data acquisition should be determined primarily for a purpose and then the flight planning should be considered in detail both in the context of laser scanner and land coverage. The flight campaign in our case was to generate LoD2 city model and has been operated with the parameters of 16 points per square meter, 80 knots flight speed, $400 \mathrm{KHz}$ reputation rate, up to $60^{\circ}$ parallel scanning pattern, $50 \%$ side overlap and $50 \%$ frontal overlap. The captured data (LAS format) then decomposed into relevant classes such as ground, vegetation and buildings. LiDAR measuring systems could be mounted on aircraft like helicopter or light aircraft. Laser scanner, like the Riegl Q680i scans the area of interest in parallel scanning lines with the help of a rotary mirror. The wavelength of the operated laser is infrared (1064 $\mathrm{nm})$ and the beam divergence in the spectrum is less than 0.5 
mrad and the scanning speed can reach up to 200 scan lines depending on the flight planning. The GPS device in the system can receive the L1 and L2 band GPS and GLONASS signals and is mounted on the tail section of the helicopter to prevent the signals from being interrupted. The 60 Megapixel digital camera of IGI DIGICAM was used to create orthophoto images. In Istanbul project, a $50 \mathrm{~mm}$ focal length lens and $8956 * 6708$ pixels colour images with pixel size of $10 \mathrm{~mm}$ were obtained. A data of 300 to $350 \mathrm{~GB}$ normally recorded for an average of 6hour flight daily.

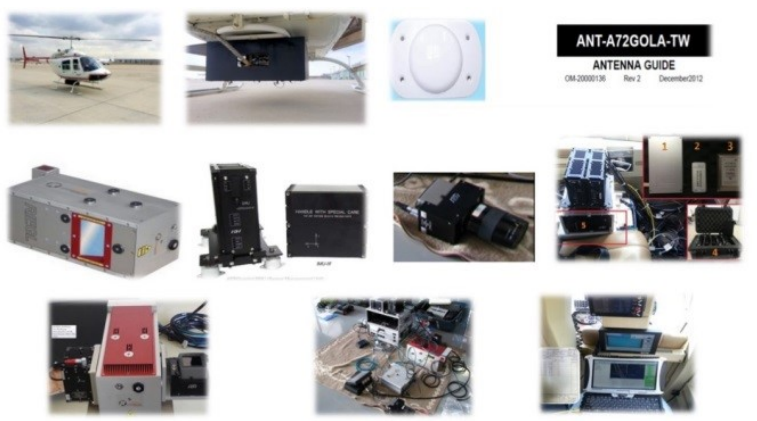

Figure 3: Data acquisition system - LiDAR

For the area of Istanbul, corresponding to about 16,000 map sheets of 1/1000 scale, adjusted and classified LAS data, digital elevation model, digital surface model and 1/1000 scale linear maps with contour lines were produced. The LiDAR data with the extension of LAS 1.2 are the data that is made up of millions of points and each is reflected from a certain object on earth surface. The classes corresponding to these points must be determined with high accuracy. Thus, the creation of models covering the desired types of detail can be provided. This classification process can be done by two methods: automatic and manual. The correct process is to automatically classify points based on an algorithm using macros. Then continue with manual control which is essential for better classification result (e.g. visual inspection by $5 \mathrm{~m}$ cross-sections via TerraScan module of TerraSolid software).

In the current map content, information about the area to be mapped, on the land, height contours, buildings, natural water resources, streams, seas, forests, under and superstructure projects, art structures, cadastral parcels are shown. This level of information content can be extracted from the LiDAR data and meets the minimum positional accuracy values expected from the map. Here, digital images taken simultaneously with the LiDAR data are also used to interpret the objects correctly.

Due to the intense construction in some regions of Istanbul or the differences in the slope of the topography, the distinction between building-vegetation and the ground-building classes have not been clearly defined in the classification of the LiDAR point cloud. Places especially of high slope of land surface, then, the distinction between the topography and the buildings close to the ground could not be classified completely, i.e. the building roofs were wrongly classified as ground class. Green colour represents vegetation and red for building structures (see Figure 4). In addition, high vegetation class, i.e. vegetation with height close to buildings creates problems during the classification stage. Thus, these problems could be solved by manual editing of the LiDAR data. Cross sections were studied in the LiDAR point cloud and the classification results were corrected by taking sections where the separation of building-topography and building-vegetation classes could not be made properly. After editing, then building vectors were created. Firstly, point cloud data were automatically classified (see Figure 4), then manual editing as illustrated in Figure 5.

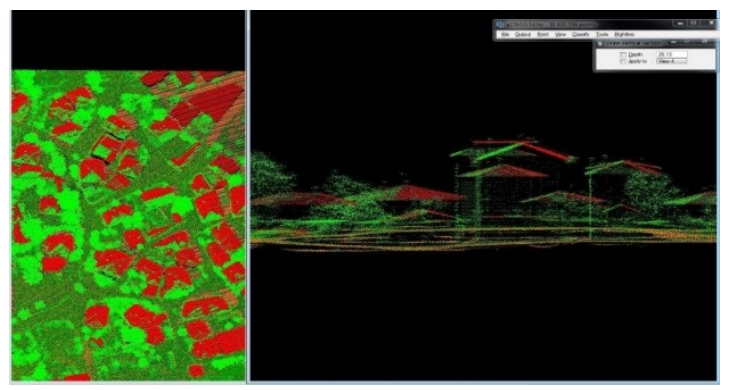

Figure 4: Situation of misclassification

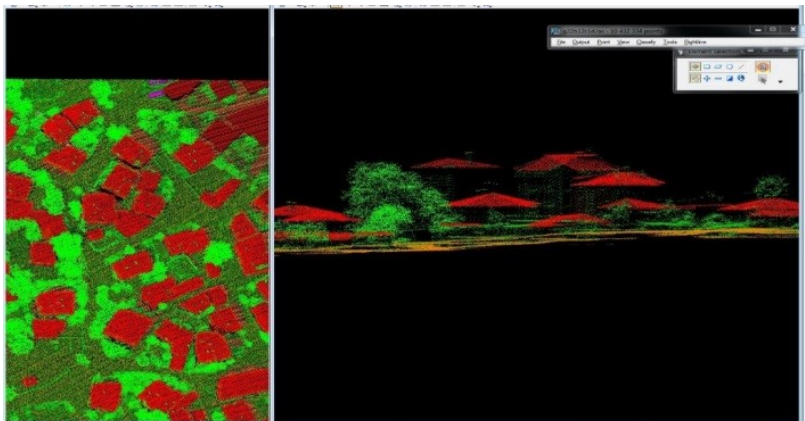

Figure 5: Corrected buildings and vegetation after manual classification

After the classification of point cloud data is done, digital elevation models (DEM), digital surface models (DSM) and only the height models such as vegetation, trees and buildings on the surface, so called normalised digital elevation models, (nDSM) are produced by the terra modeller software module. The DSM product is mainly used as the basic data in determining trees heights, trunk diameters and timber volumes in forest management.

Building vectors were created by using building photographs and existing data. The outline of the buildings was based on polygons of the building exterior. Missing and defective buildings are corrected by taking advantage of the aerial photographs. Below are examples of building solid models in the LoD2 model produced in DGN format, see Figure 6 and Figure 7.
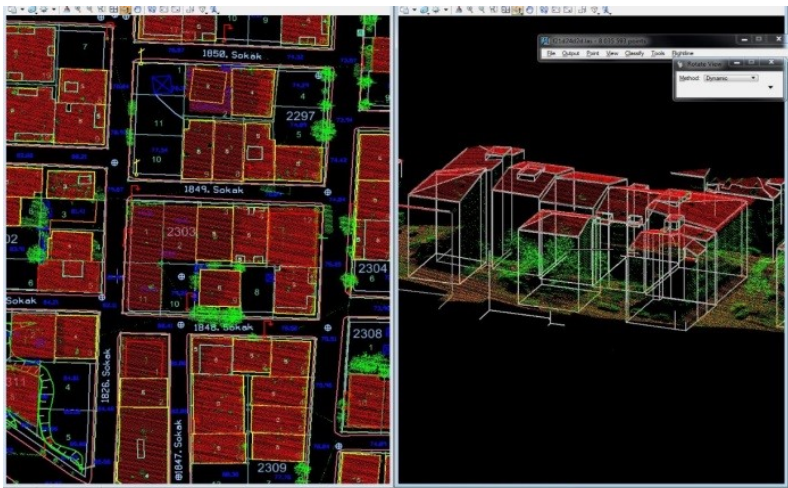
Figure 6: Buildings in DGN format
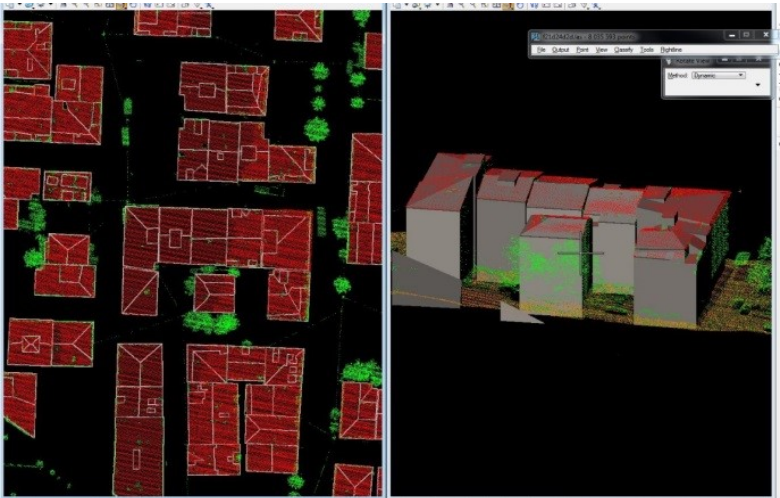

Figure 7: Solid buildings model

In the production of $3 \mathrm{D}$ city model, the automatic production of the building models of the roofs with smooth structure gives acceptable results, however, the roofs with mixed roof surfaces such as the domes, the cones, other mosque structures, etc. have some limited outcomes. These complex structures were manually modelled, see Figure 8.
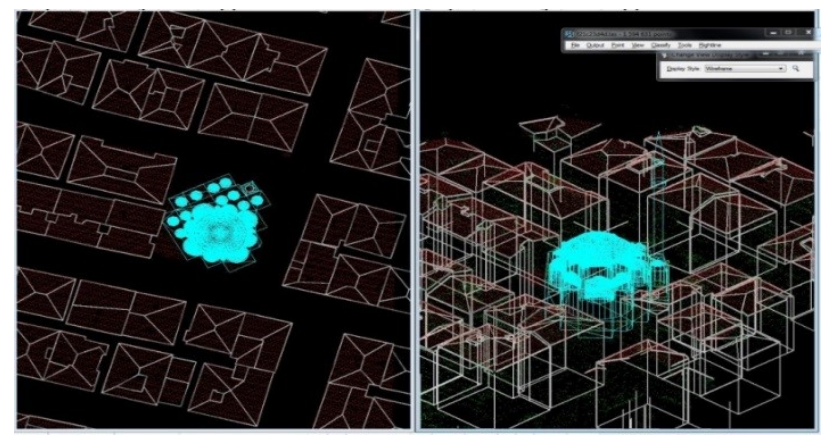

Figure 8: Manual digitizing of complex building

Figure 9 and 10 illustrate the model of the city model created from manual drawings and editing of the roofs.

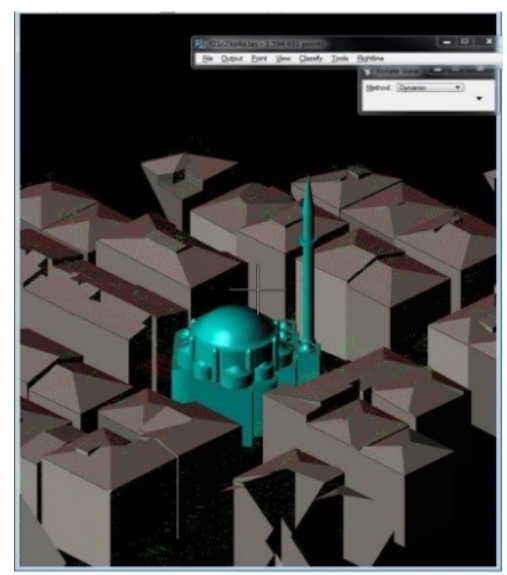

Figure 9: A mosque in LoD2 model (via manual technique)

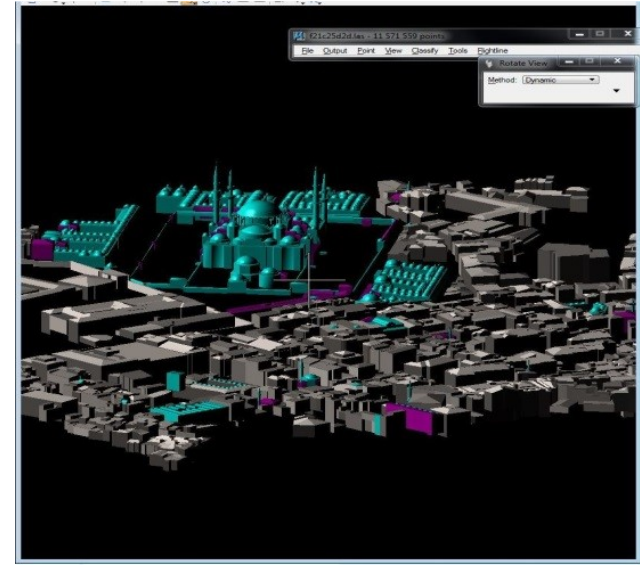

Figure 10: Complex (mosques) objects via manual technique

\subsection{Mobile and Panoramic Imagery Data Collection}

The system consists of four major units, they are panoramic camera, IMU, GPS and LiDAR components. The camera is from NcTech company, UK. It has equi-rectangular projection $\left(360^{\circ}\right.$ by $180^{\circ}$ ) with the image size of $13850 \times 6925(95.9 \mathrm{MB})$ pixels where the ground resolution of panoramic images is about $1 \mathrm{~cm}$ at $20 \mathrm{~m}$ object distance. The IMU with Applanix POS LV 520, GPS with Trimble BD982, and LiDAR with Velodyne VLP-16. The following figures show the integrated system of the employed panoramic data collection (see Figure 11).
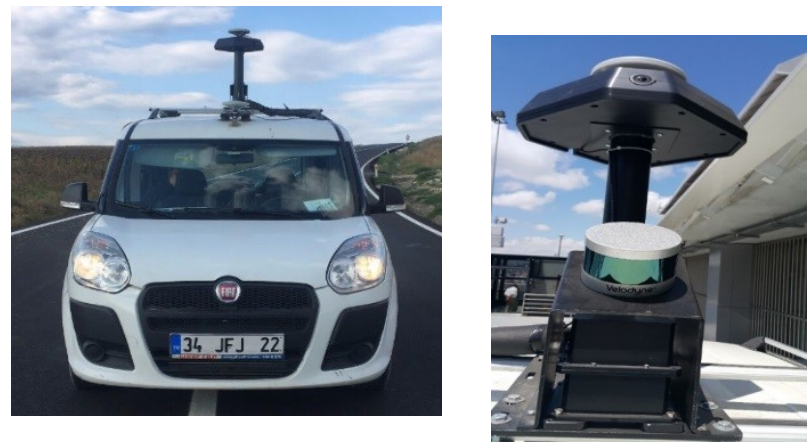

Figure 11: Mobile mapping system

The system is meant to complement the existing airborne acquisition one especially for areas that the later technique could not cover. One of the advantages - able to capture texture of buildings facades and other important objects. 


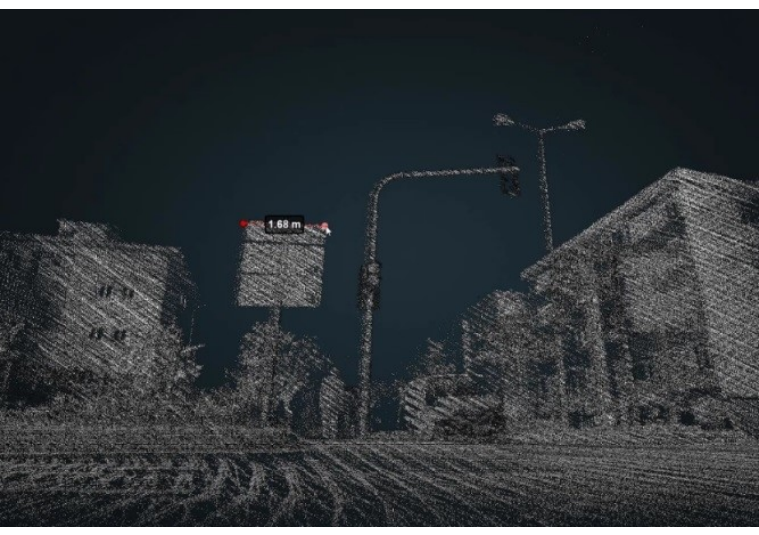

Figure 12: Points cloud of the buildings and other features

The above figure (Figure 12) shows the points cloud from mobile mapping system and below shows the points cloud with CAD data (Figure 13 and Figure 14).

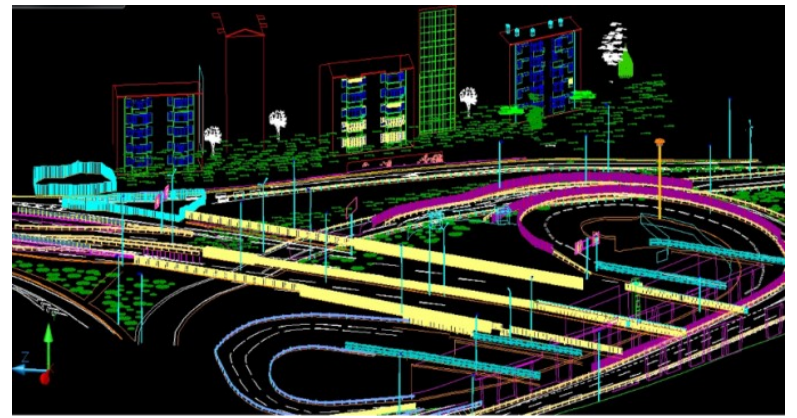

Figure 13: 3D CAD data derived from mobile scanning

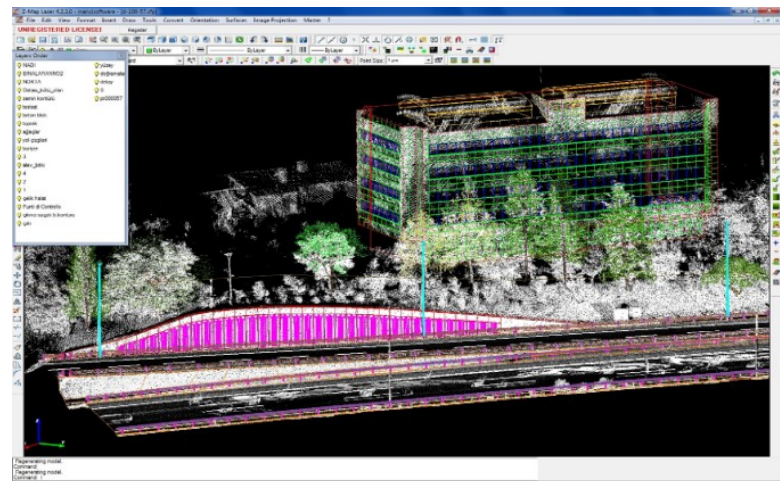

Figure 14: Point clouds with CAD data.

The collected data from the system were used for creating 3D models of objects in the area especially buildings and other streets furniture as illustrated in the above figures. One major point to note that this technique provides fast and accurate datasets, however, semi or manual editing of the data is common task.

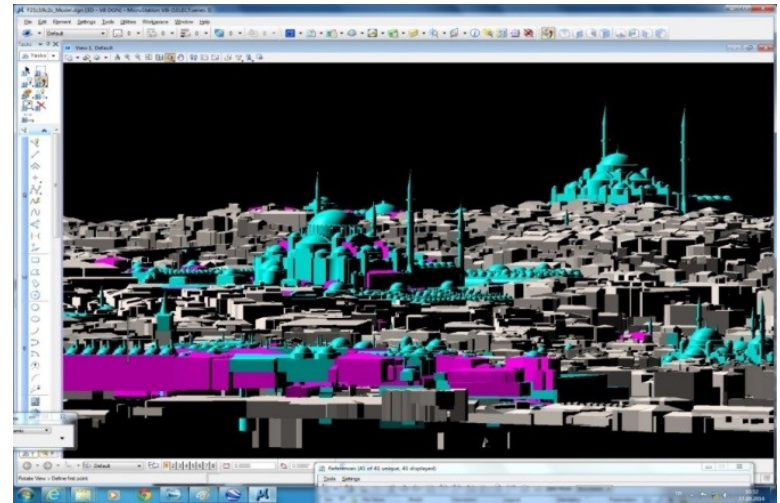

Figure 15: A part of Istanbul LoD2 city models with 3D mosques and surroundings

The above figure (Figure 15) shows LoD2 buildings and several important landmarks like mosques (3200 mosques) in LoD2+ (means they were created with details geometry). The area covers approximately street distance of $35,000 \mathrm{~km}$ for the outdoor street mapping. Trees were not modelled but represented only with points cloud.

\subsection{Backpack system}

As for the panoramic data acquisition, we utilise backpack system that consists of panoramic camera, IMU, and GPS (for outdoor). The technique is meant for areas with narrow streets and areas that inaccessible by the van, and places where GPS does not work properly. The system as illustrated in Figure 16 mounts at the back of a person for data acquisition.

Figure 16 :

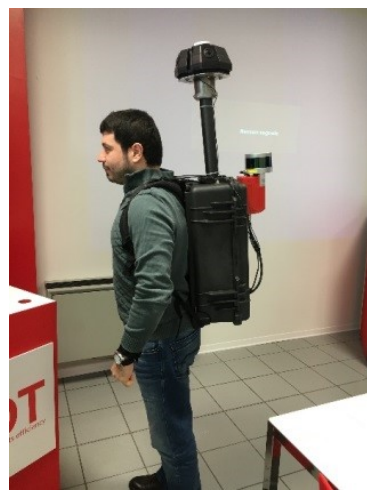

system

\subsection{Panoramic Web Application Services}

The panoramic images were utilised for the development of Web application services (i.e. similar as Google Street View) with several modules such as measurements and other geometrical tools. The following figure shows the panoramic image sample (Figure 17). 


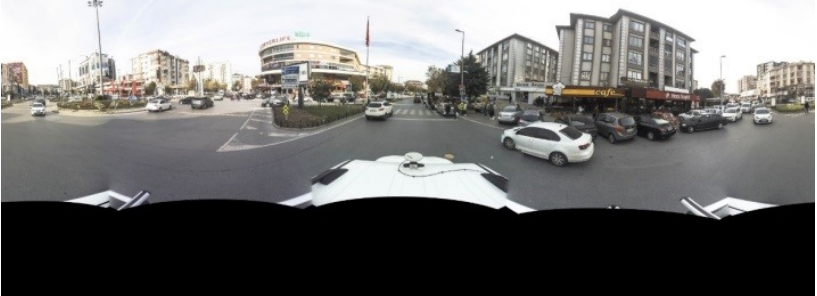

Figure 17: Sample panorama from a Istanbul street

The resulting panoramic images and auxiliary data will be integrated into the client's servers and Web-based services such as the municipal city guide in accordance with their specifications. This city "streetview" application data can be shown on mobile platforms (Android and IOS). $360^{\circ}$ panoramic images can be seen through VR glasses. At the end of all virtual tours and point clouds datasets can be published a web-based platform. This provides online and offline visualization of the data through a server and several measurement tools (building heights, street distances, etc), see Figure 18. This large panoramic dataset provides and empower many aspects of smart city requirements.

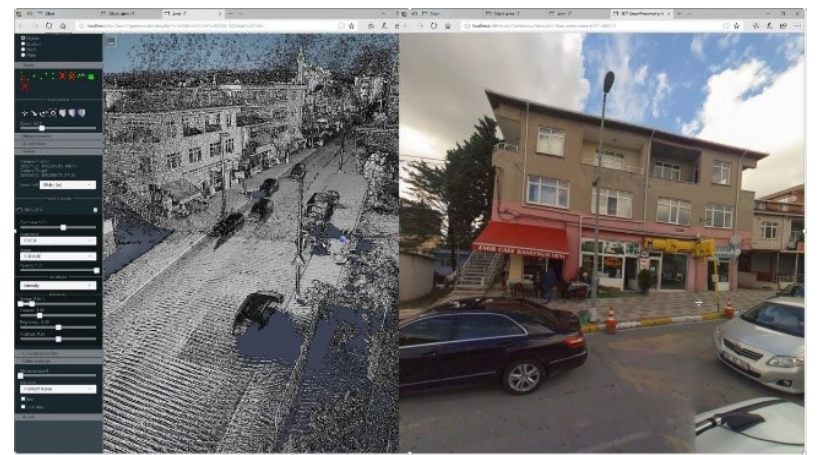

Figure 18: The combined view of points cloud and panoramic imagery of same location in Istanbul

\subsection{LoD3 Buildings Generation}

The LoD3 buildings were generated mainly for major landmarks and historical ones especially in the Historic Peninsula of Istanbul (approximately more than 1000 buildings). Each comes with textures and geometries, examples of LoD3 buildings as in the following Figure 19 (a), (b), (c), and (d).

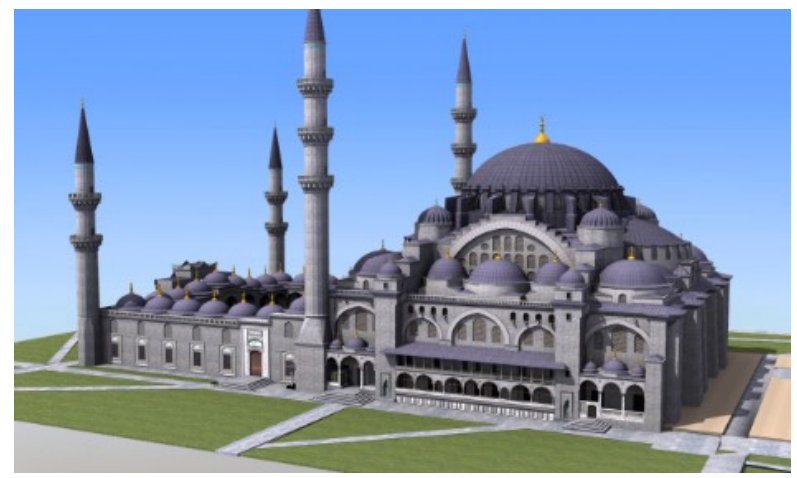

Figure 19 (a)

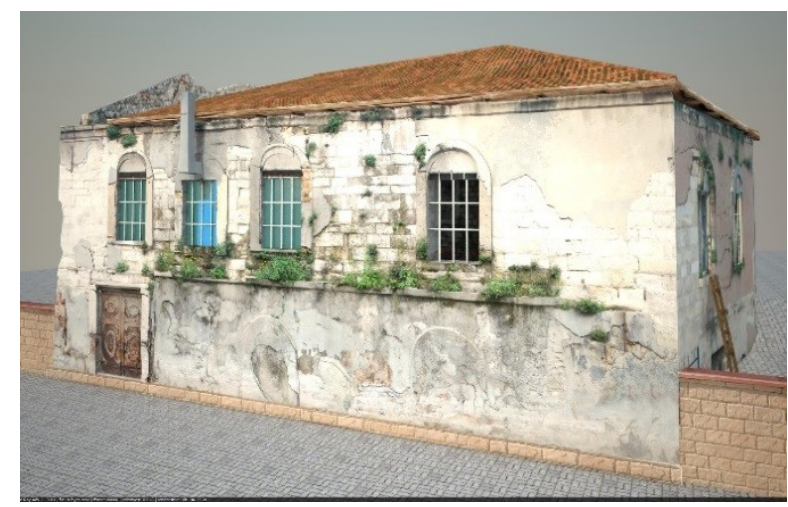

Figure 19 (b)

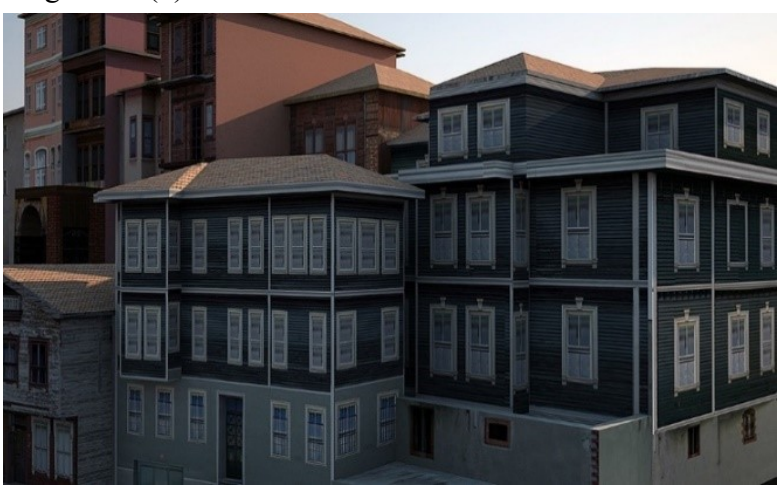

Figure 19 (c)

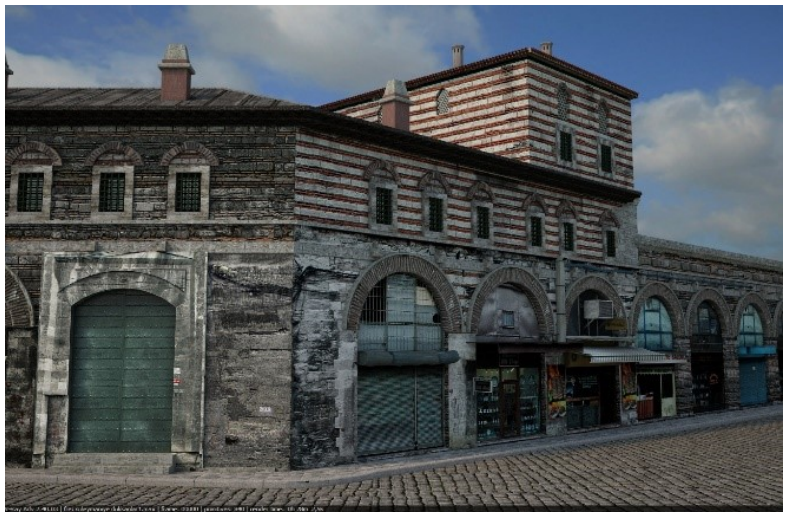

Figure $19(d)$

The geometry comes from 3D points cloud and digitized by using 3D CAD software. 520 buildings of Fatih historical area were modelled according to LoD3 and based on architectural plans and high resolutions images of facades. 3D CAD models (.DGN format) were created from airborne LiDAR points cloud data and high resolution images were imported into 3D StudioMax environment, then the respective files were stored in 3DS file format and stored in the 3D server. Other objects like monuments of 345 in total were created as of LoD3+. For this project, models were created from terrestrial laser scanning of indoor and outdoor scans with $\mathrm{mm}$ precision. 3D drawings were generated using point cloud by using Zmap software which is working on Autodesk platform. 


\subsection{LoD4 Buildings Generation}

LoD4 buildings model reveals many details of the interior e.g. major landmarks and important buildings. It is the highest details within CityGML schema (Kolbe, 2010). LoD4 is an LoD3 upgraded with interior. The LoD of external geometry and the semantics remain equal. In general, not all buildings in a city will be in this category due to huge development costs. Istanbul city developed several buildings as LoD4 based on indoor laser scanning. Figure 20 shows the points cloud of the indoor space with its corresponding generated geometry in CAD. The 3D points cloud of indoor (see Figure 19) and the solid modelling within 3D StudioMax (as illustrated in Figure 20).

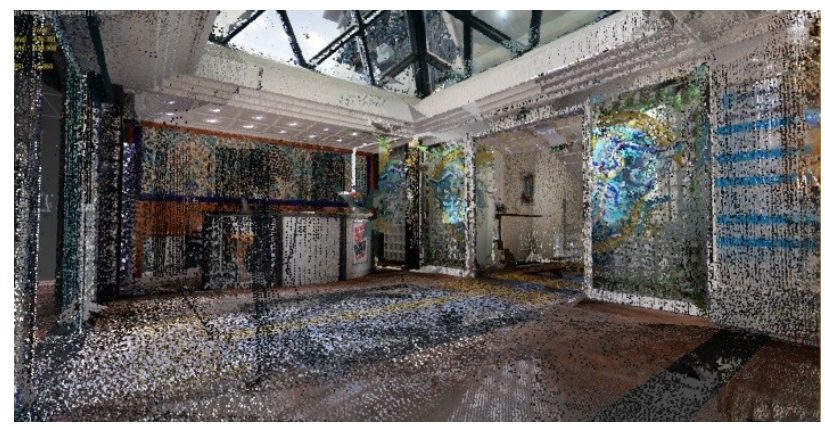

Figure 20: The points cloud of the indoor

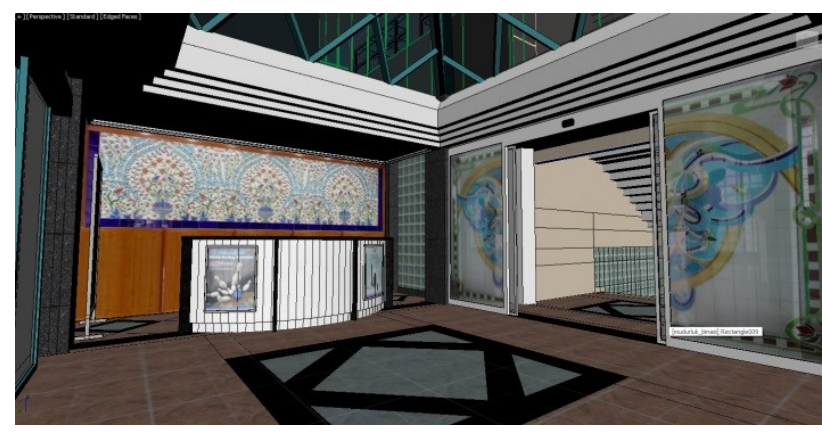

Figure 21: The 3D solid modelling - LoD4

More details explanation of the whole process of creating 3D city model for the Istanbul city as illustrated in the workflow in Appendix A.

\section{THE COMPLETE WORKFLOW}

The 3D city modelling workflow could be described as follow:

Airborne LiDAR data acquisition via Riegl Q680i laser scanner, IGI DigiCam 60, IGI Areo Control SMU, and CCNSS systems. The points cloud then need to be geometrically corrected in terms of relative and absolute accuracy by using Riegl RiProcess and RiAnalysis modules. Next, is the classification of the points cloud by using MicroStation V8i Connect, TerraSolid and TerraScan modules. The automatic classification produces $90 \%$ accuracy. Problems of the classification normally occur around buildings close to high vegetation (high trees). However, manual classification improves the classification outcomes. Then, generation of DEM, DSM, nDSM (normalised DSM) with 0.25 $\mathrm{m}$ GSD. Visual check of the outcomes is required, export to "3D
Server" if meet the specification, otherwise, need to recheck the classification phase.

Next, automatically generation of 3D building with roofs by using TerraModeler and also footprints from 1:1000 basemaps as layer for 2D map. The vectorization of buildings work for normal facades, but more complex objects like mosques, tombs, etc require manual works. Then, export the 3D CAD buildings (DGN and DWG files) to the server. The outcome of this phase is LoD3+ buildings.

Next, the CAD data (DGN and DWG files) transform to BLOB.xml by using FME tools. The BLOB files then utilised to create LoD2 and LoD3 buildings with FME. At this stage, CityGRID checks and creates topologically correct objects outer boundaries, general rooflines, vertical roof facades, and floors are among the objects. Semantics information of the buildings are also available, and finally export to the "3D Server".

Appendix A illustrates the complete workflow.

\section{SOME ISSUES AND CHALLENGES}

\subsection{Modelling of roofs}

In Istanbul most footprints maps are based on aerial photography mapping where roofs edges were considered as part of building footprints. The following figure shows the typical situation of roofs and footprints (i.e. "fat" building). The situation creates very limited texture of building facades. Thus, terrestrial laser scanning could be utilised for mapping of the facades. Classification of roofs and vegetation has some limitations where some situations of these two objects could not be automatically solved. Thus, manual classification resolves the issue at incomplete and "noisy" building-vegetation places.

\subsection{LoD2 to LoD3 upgrading}

LoD3 buildings have roofs and other structures such as facades both with geometry and semantic information. The facades could be mapped with terrestrial laser scanning technique. The transformation from LoD2 to LoD3 is not straight forward, it involves semi and manual editing. Important landmarks like mosques - we have detailed models (LoD2.3) but without texture. Status wise - out of 1.5 million buildings ( 3800 buildings as LoD3). Generation of LoD3 buildings are quite straight forward for simple buildings, however, not so for complex buildings like mosques, bridges, historical walls, monuments, etc. At the moment, the LoD3 models are based on CityGRID XML format. Conversion to full CityGML structure is the next task. Expanding LoDs towards CityGML 3.0 as proposed by Biljecki (2016) is our next task.

\subsection{Management of large datasets}

The data collection creates huge datasets, around 1.5 million buildings cover an area of $5400 \mathrm{~km}^{2}$. The data consumes approximately $25 \mathrm{~GB}$ per $\mathrm{km}^{2}$, thus $135,000 \mathrm{~GB}(135 \mathrm{~TB})$ of storage for whole city. More data will be populated in the server once the Backpack mapping system in action. The city has many small alleys and inaccessible by mapping vehicle. Buildings and facades from these narrow streets will be captured by Backpack scanning system. The panoramic data collection covers 32,000 $\mathrm{km}$ (for the whole Istanbul) and consumes $70 \mathrm{~TB}$ data per district 
and Istanbul covers 39 districts, thus, in total of $2730 \mathrm{~TB}$ or 2.73 PB of data. All data are stored in the Server and can be accessed via local network with some restrictions. This configuration has some drawbacks e.g. data could not be properly managed as database. The following section (5.4) describes the general view of the planned database schema.

\subsection{Geospatial database}

This is very important component of any geospatial based information system including 3D city model, that is to provide queries and answers to any questions related to any objects within the city. Management, analyse, and visualization of the CityGML based objects could be made possible with this spatial database. Currently, all the objects are stored as flat files in a server. Proper geospatial database is the near-future plan. One of the possible options is via 3DCityDB Webclient (open source) within Oracle Spatial (or PostgresSQL or PostGIS) that utilises CityGML schema definition files and Cesium Virtual Globe. 3DCityDB is CityGML compliant. Figure 22 shows the proposed system architecture for better management of data and applications.

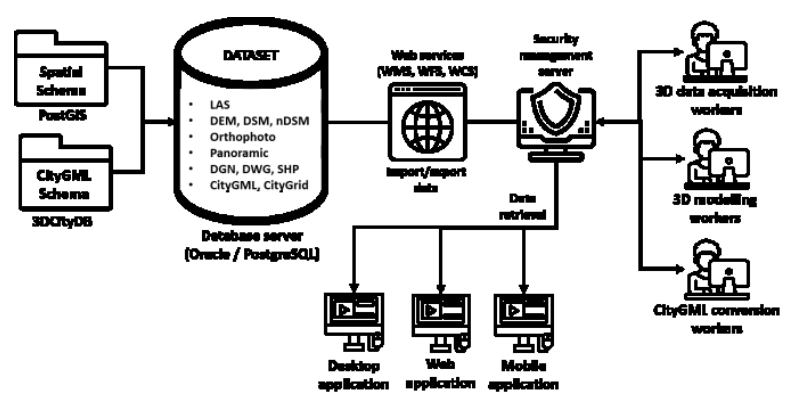

Figure 22: The system architecture for spatial database and other sub-systems components

\subsection{Software and tools limitation}

Automatic classification module (MicroStation TerraScan) faces some limitations for complex situations and buildings, especially objects located closed to high vegetations (trees), thus, manual operation applies.

\subsection{System and human errors}

System calibration errors may result inaccurate data acquisition. Other constraints such as weather conditions, thus time delay and time consuming. Data collection planning also important and need to be addressed prior to data acquisition deployment.

Boresight calibration problems between units (IMU, GPS, Camera) has to be addressed properly since it directly affects the data accuracy. In general, human errors also contribute to the performance and outcome e.g. missing parameters related to the systems, missed coding, incomplete road survey, etc.

\section{CONCLUSIONS AND OUTLOOK}

We have described the development of 3D city model for the Istanbul city that based on LiDAR and panoramic images datasets. Several data acquisition, processing, and modelling tools were utilised. Our experience shows that acquiring, processing, modelling and developing 3D objects for large city like Istanbul demand huge efforts both in the aspects of human resource, hardware and software. Complex and other irregular objects such as mosques require manual operations (editing, classifications). Automatic tools face some limitations for complex objects. Some limitations, issues and challenges of the current tools have been highlighted.

In future, we would like to explore UAV (unmanned aerial vehicle) as part of data acquisition technique especially for small areas with details and quick outcomes. Forrest areas and less populated areas could be complemented by the UAV system. Backpack mapping system could be an option for areas that inaccessible by the mobile LiDAR system since Istanbul city covered by many narrow roads and alleys.

\section{REFERENCES}

Biljecki, F. (2017). Level of Details in 3D city models. PhD thesis, TU Delft, the Netherlands, $353 \mathrm{p}$.

Biljecki,F. J. Zhao, J. Stoter, H. Ledoux (2013). Revisiting the Concept of Level Of Detail in 3D City Modelling, Chapter in: ISPRS Annals Volume II-2/W1, Proceedings of the ISPRS 8th 3D GeoInfo Conference \& WG II/2 Workshop (Ü. Işıkdağ, ed.), Istanbul, pp. 63-74, 2013.

Biljecki, F., H. Ledoux, J. Stoter, J. Zhao (2014). Formalisation of the level of detail in 3D city modelling, In: Computers, Environment and Urban Systems, 48, pp. 1-15, 2014.

Buyuksalih, G., Bayburt, S., Baskaraca, P., Buyuksalih, I., and A. Abdul-Rahman (2018). 3D city modelling of Istanbul city based on LiDAR data and panoramic images - issues and challenges. Tutorial at GGT 2018 International Conference, Kuala Lumpur, Malaysia

Kolbe, T. (2017). CityGML goes to Broadway. Photogrammetric Week 2017, Stuttgart, Germany.

Kolbe, T. (2010). Tutorial on 3D city modelling and CityGML. 3D GIS Lab, Universiti Teknologi Malaysia, Johor Bahru, Malaysia.

Khoo, V., and B. K. Soon (2016). Singapore 3D. Bentley Infrastructure Conference. London, U.K.

OGC (2018). CityGML Standard.

http://www.opengeospatial.org/standards/citygml

OGC (2012). OGC City Geography Markup Language (CityGML) Encoding Standard.

Prandi, F., F. Devigili, M. Soave, U. Di Staso, and E. De Amicis (2015). 3D Web visualization of huge CityGML models. ISPRS Archives Vol. XL-3W3, pp. 601-605

Stoter, J. (2016). 3D city modelling tutorial. TU Delft, The Netherlands.

Stoter, J., L. van den Brink, G. Vosselman, J. Goos, S. Zlatanova, E. Verbree, R. Klooster, L. van Berlo, G. Vestjens, M. Reuvers, S. Thorn (2011). A generic approach for 3D SDI in the Netherlands, In: Proceedings of the Joint ISPRS Workshop on 
The International Archives of the Photogrammetry, Remote Sensing and Spatial Information Sciences, Volume XLII-4/W12, 2019 5th International Conference on Geoinformation Science - GeoAdvances 2018, 10-11 October 2018, Casablanca, Morocco

3D City Modelling \& Applications and the 6th 3D GeoInfo Conference, Wuhan, pp. 22, 2011. 
Appendix A

The complete workflow of the 3D city modelling of Istanbul

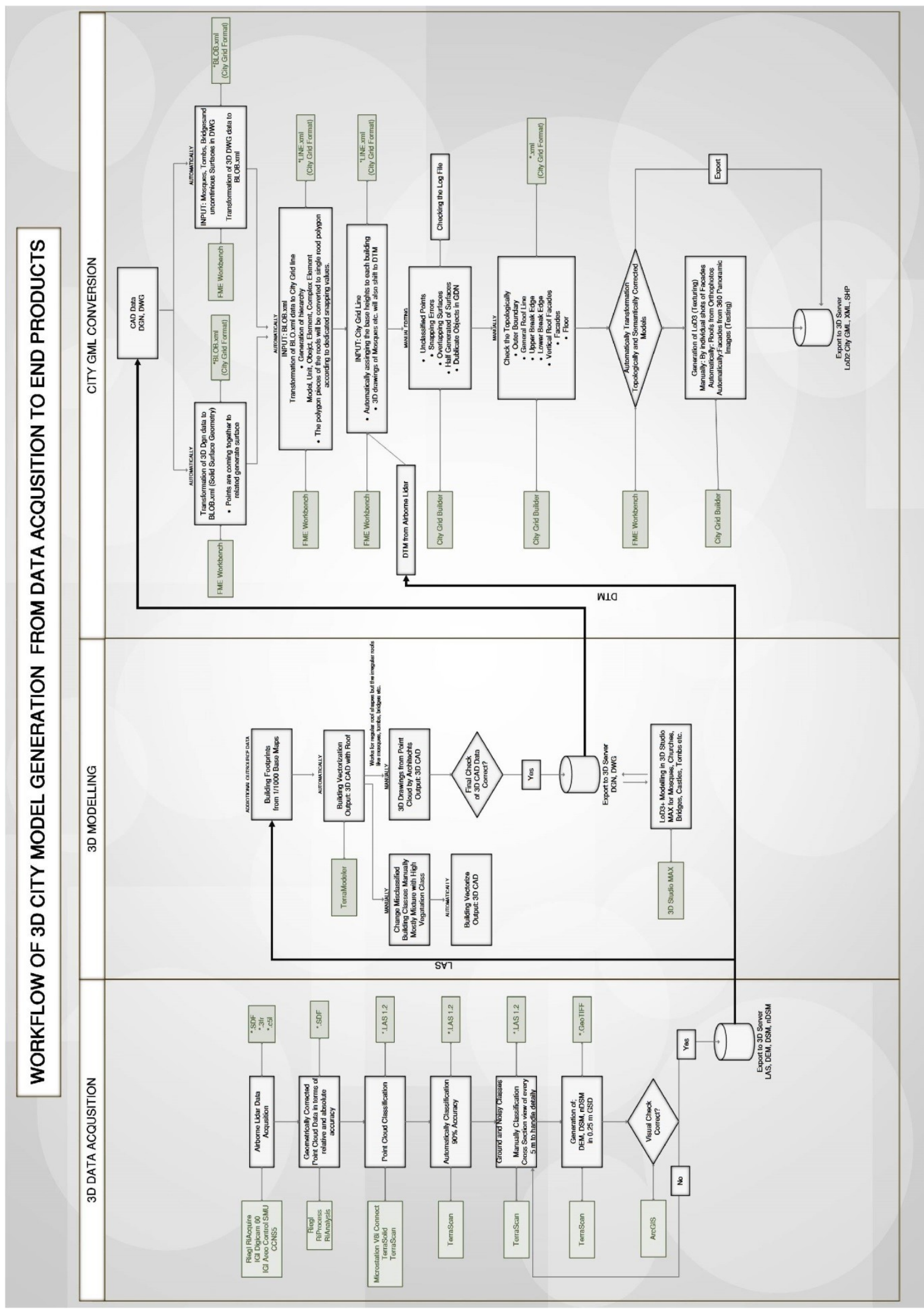

